\title{
Fireball Multi Object Spectrograph: As-built optic performances
}

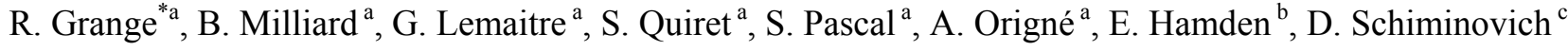 \\ ${ }^{a}$ Aix-Marseille Université, CNRS, LAM (Lab. d'Astrophysique de Marseille) UMR 7326, 13388, Marseille, France \\ ${ }^{\mathrm{b}}$ Californian Institute of Technology, 1200 E California Blvd, Pasadena, CA 91125, USA \\ ${ }^{c}$ Department of Astronomy, Columbia University, New York, NY 10027, USA
}

\begin{abstract}
Fireball (Faint Intergalactic Redshifted Emission Balloon) is a NASA/CNES balloon-borne experiment to study the faint diffuse circumgalactic medium from the line emissions in the ultraviolet $(200 \mathrm{~nm})$ above $37 \mathrm{~km}$ flight altitude. Fireball relies on a Multi Object Spectrograph (MOS) that takes full advantage of the new high QE, low noise $13 \mu \mathrm{m}$ pixels UV EMCCD. The MOS is fed by a 1 meter diameter parabola with an extended field $\left(1000 \operatorname{arcmin}^{2}\right)$ using a highly aspherized two mirror corrector. All the optical train is working at $F / 2.5$ to maintain a high signal to noise ratio. The spectrograph ( $\mathrm{R} \sim 2200$ and 1.5 arcsec FWHM) is based on two identical Schmidt systems acting as collimator and camera sharing a $2400 \mathrm{~g} / \mathrm{mm}$ aspherized reflective Schmidt grating. This grating is manufactured from active optics methods by double replication technique of a metal deformable matrix whose active clear aperture is built-in to a rigid elliptical contour. The payload and gondola are presently under integration at LAM. We will present the alignment procedure and the as-built optic performances of the Fireball instrument.
\end{abstract}

Keywords: Spectrograph, Ultraviolet, Multi-object spectroscopy.

\section{INTRODUCTION}

Fireball (Faint Intergalactic Redshifted Emission BALLoon) is a NASA/CNES balloon-borne experiment to study the faint diffuse circumgalactic medium from line emission in the ultraviolet $(200 \mathrm{~nm})$ above $37 \mathrm{~km}$ flight altitude. Fireball is a large stratospheric gondola ( 5 meters and 1.6 metric Tons) with a 1 meter pupil diameter fixed parabola mirror fed by a 1.3 meters siderostat mirror with a guidance accuracy of less than 1 arcsec RMS in the new version ${ }^{1}$.

Fireball has flown twice in 2007 and 2009 from Palestine (Texas) and Fort Sumner (New Mexico) respectively. The sensitivity of the previous instrument, ${ }^{2}$ based on an Integral Field Unit (IFU) with a 400 UV fiber bundle and a photocathode photon counting detector, was not sufficient to detect the faint diffuse emission of the cosmic web. The design of the new payload adopts two major changes. First, a low noise L3 CCD detector from E2V enhanced in UV $(200 \mathrm{~nm})$ by a proprietary delta doped process from Caltech/JPL and a multilayer anti-reflection coating. The typical improvement is a factor 8 over the previous photocathode detector. Second, instead of a detailed analysis of a few targets during the flight with an IFU, we decided to rely on a Multi Object Spectrograph (MOS) with the capability of 50 slits on a mask giving access typically to 150 objects for a 7 hours flight.

The optical design of this MOS has been described in a 2014 SPIE paper $^{3}$ and we will present hereafter the alignment procedure and the as built performances of the Fireball optical train.

\section{THE UNIT MAGNIFICATION TWO MIRROR FIELD CORRECTOR}

The MOS requires a much larger corrected field of view (FOV) than the previous IFU (1000 versus $16 \operatorname{arcmin}^{2}$ ). While the IFU could rely on the small FOV of the F/2.5 parabola, the MOS needs a field corrector working at unit magnification to keep the $\mathrm{F} / 2.5$ aperture ratio. This leads to fast and highly aspherized mirrors and consequently to a challenging manufacturing process. Indeed, the mirrors are working at F/0.8 and their main characteristics, in particular their departures from the best sphere, are summarized in the table 1 .

Space Telescopes and Instrumentation 2016: Ultraviolet to Gamma Ray, edited by

Jan-Willem A. den Herder, Tadayuki Takahashi, Marshall Bautz, Proc. of SPIE Vol. 9905, 990531

(C) 2016 SPIE - CCC code: 0277-786X/16/\$18 - doi: 10.1117/12.2233267

Proc. of SPIE Vol. $9905990531-1$ 


\begin{tabular}{l|l|l} 
& Focal Corrector 1 & Focal Corrector 2 \\
\hline Blank Material & Zerodur & Zerodur \\
\hline Diameter & $85 \mathrm{~mm}$ & $285 \mathrm{~mm}$ \\
\hline Thickness at center & $20 \mathrm{~mm}$ & $10 \mathrm{~mm}$ \\
\hline Radius of curvature & $133.96 \mathrm{~mm}$ & $277.16 \mathrm{~mm}$ \\
\hline Eccentricity & -2.527 & -0.198 \\
\hline Departure from best sphere & $0.08 \mathrm{~mm}$ & $0.126 \mathrm{~mm}$ \\
\hline Surface error (RMS) & $61 \mathrm{~nm}$ & $97 \mathrm{~nm}$ \\
\hline Roughness (RMS) & $0.22 \mathrm{~nm}$ & $0.679 \mathrm{~nm}$
\end{tabular}

Table 1: Main characteristics of the as-built focal corrector mirrors

The mirrors have been ground very close to the final shape using $\mathrm{CNC}$ 5-axis machine. The surfaces have been then polished using computer controlled polishing robots and measured by interferometry. All the processes for such deep aspheres have been conducted on deterministic machines available at WINLIGHT OPTICS for the manufacture of high performance aspheres.

The form error characterization has been measured with a Luphos 3D non-contact interferometric system well adapted to measure deep aspheres. The Luphos analysis for the two mirrors are presented in figure 1.

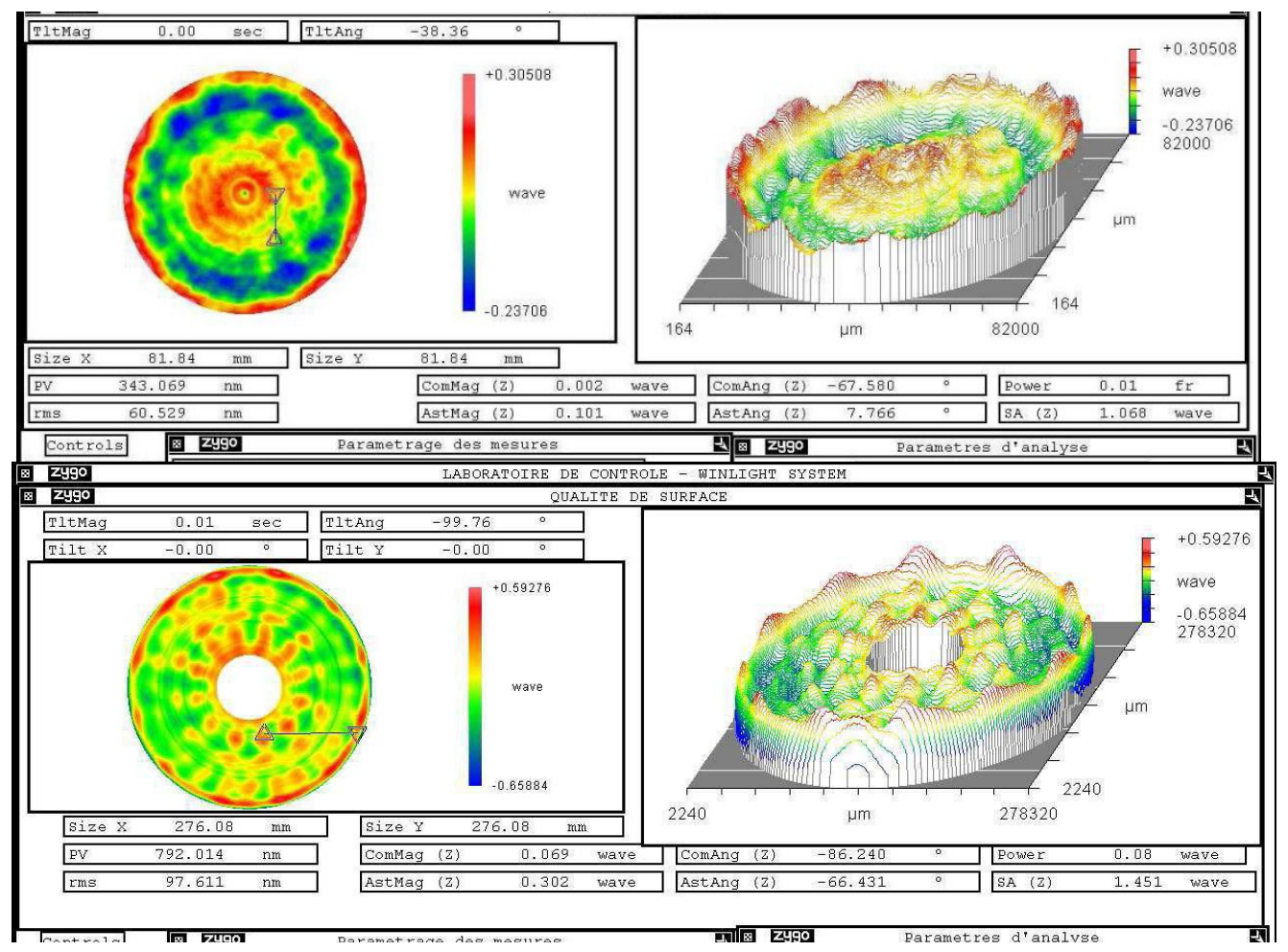

Figure1: Form error of the Focal Corrector 1 (top) and Focal Corrector 2 (bottom) measured with a LUPHOSCAN.

We performed a detailed sensitivity analysis and deduced alignment tolerances with the assumption of a degradation of the nominal PSF by less than $10 \%$. The main tolerances are summarized in Table 2 . This analysis shows that the small convex mirror has to be aligned with 15 micrometers in centering and 1 arcmin in tilt relative to the large concave. To achieve these tight alignment requirements, we designed and built a dedicated optical bench (Figure 2 left) to align the 
focal corrector in front of an interferometer in double pass. In a second step, we crosschecked this interferometric test with a single pass optical test and measure the PSF. The convex mirror mount is attached to a stack of stages (Figure 2 right) to provide five degrees of freedom. Once the best PSF was reached we bonded the convex mirror mount via a tripod to the large concave mirror mount staying fixed and referenced to the bench. This method often called "liquid shim" has the advantage of not adding stress on the optics via their mounts.

\begin{tabular}{l|l|l|l} 
& Decenter & Tilt & Focus \\
\hline Field lens & $\pm 1 \mathrm{~mm}$ & $\pm 0.9^{\circ}$ & $\pm 500 \mathrm{um}$ \\
\hline Focal corrector 1 (small) & $\pm 15 \mathrm{um}$ & \pm 1 arcmin & $\pm 30 \mathrm{um}$ \\
\hline Focal corrector 2 & reference & reference & reference
\end{tabular}

Table 2: Focal corrector alignment tolerances
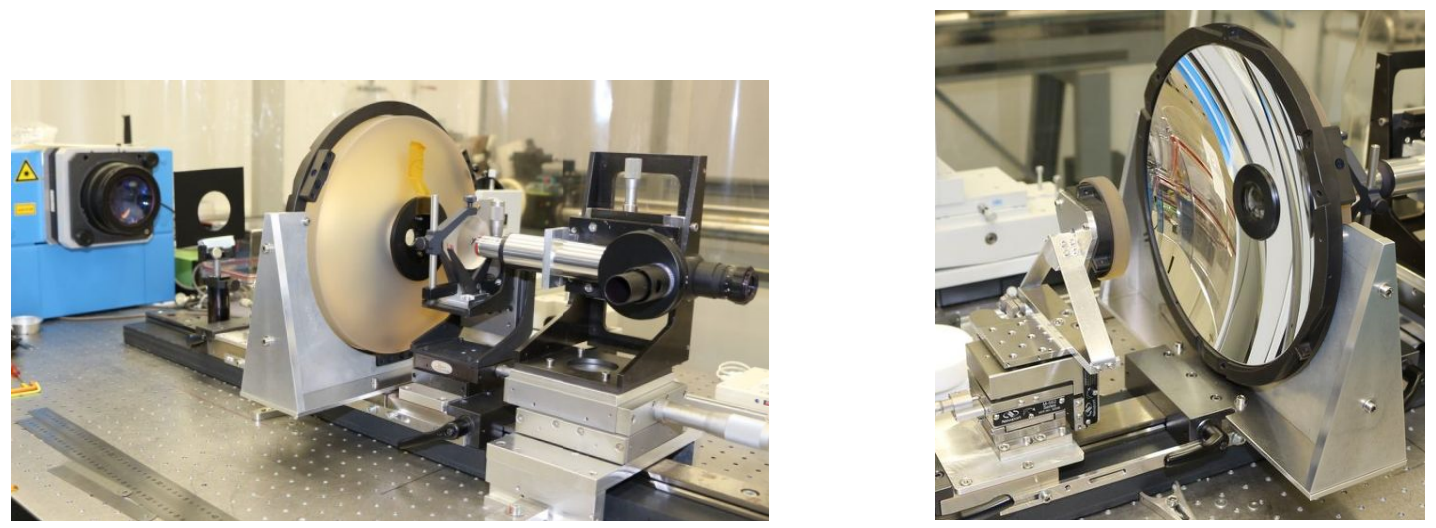

Figure 2: Dedicated optical bench to align the Focal Corrector (left). The convex is attached to a stack of stages providing five degrees of freedom allowing alignment and bonding (right)

A 5 micrometers pinhole at the entrance of the focal corrector is imaged on a visible CCD allowing the computation of the encircled energy curve (Figure 3). We can see that $80 \%$ of the incident energy will go through the 50 micrometers slit width.
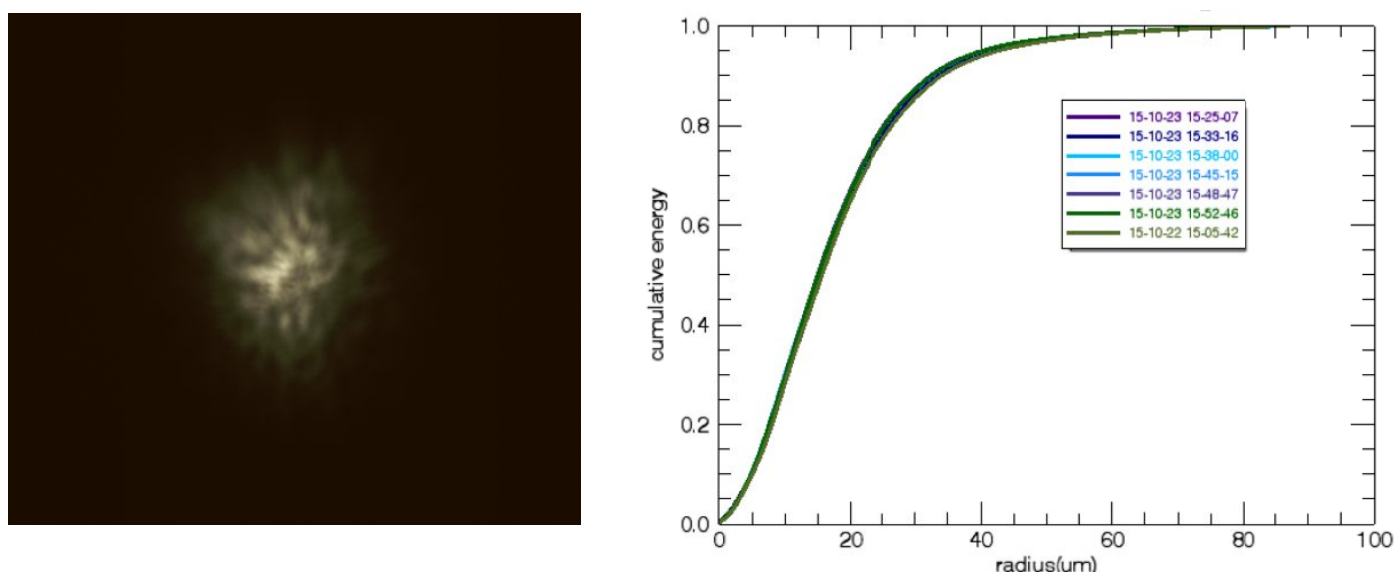

Figure 3: (left) Image of a $5 \mu \mathrm{m}$ pinhole and the entrance of the Focal corrector and (right) the corresponding Encircled Energy function 


\section{THE SCHMIDT COLLIMATOR AND CAMERA}

Compared to the focal corrector, the manufacturing of the collimator and Schmidt camera mirrors are much simpler, relying on two spherical mirrors and two folding flats.

The collimator optics (first two mirrors) have been coated by Cascade Optical with a notched filter centered on the 200 $\mathrm{nm}$ Fireball bandpass. Knowing that the UV CCD is also sensitive in the visible, a coating is deposited prior to the UV notch filter, to absorb at visible wavelengths, giving the black aspect shown in Figure 4. Doing this filtering on the two first mirrors before the light reaches the grating help to reduce the amount of visible light that the grating could diffuse in the spectrograph housing and later, onto the detector.

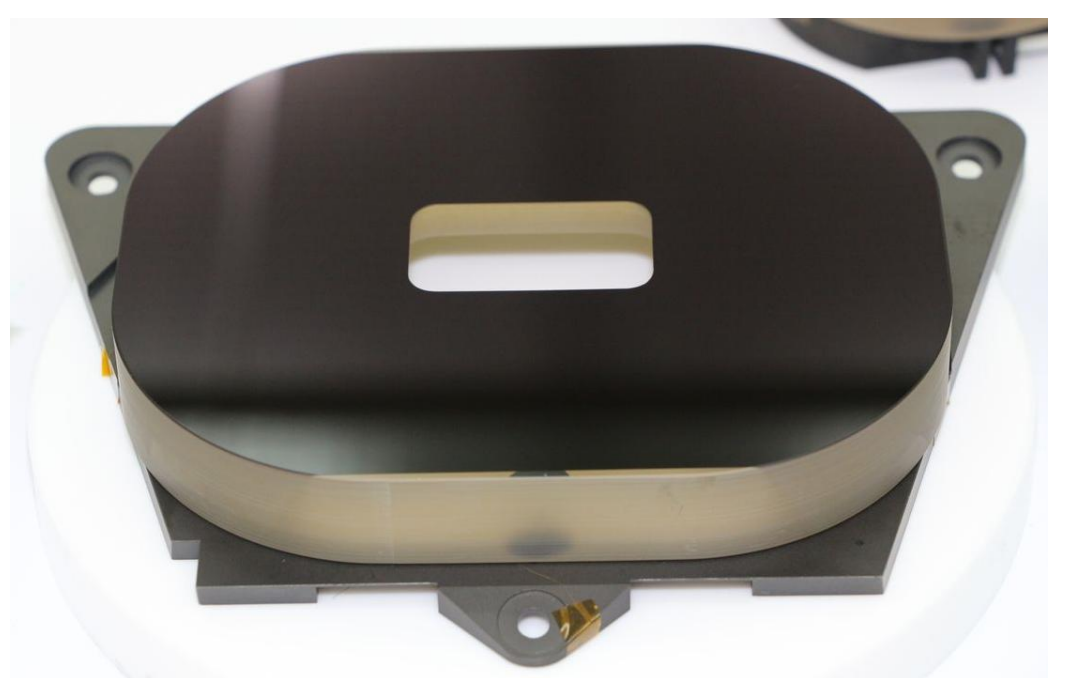

Figure 4: Flat folding mirror coated with an UV notched filter plus a visible rejection

For the spectrograph optical train the alignment tolerances are much looser than for the two mirror focal corrector (Table 3 ). We then rely on mechanical shims to adjust each optic at their theoretical position by doing a Faro arm metrology loop. In this metrology we shortcut the optic mounts by directly measuring the optical surfaces on the very edge and in the center of mirrors where obscuration occurs anyway. We then exported the coordinate data set in Catia 3D CAO software to determine the new set of shims; typically the loop converges in two steps. In the case where a tip/tilt default is found we used spherical washers under the 3 points of fixation to the bench to avoid mount deformations.

\begin{tabular}{c|c|c|c} 
& Decenter & Tilt & Focus \\
\hline Schmidt Mirror 1 & $\pm 100 \mathrm{um}$ & $\pm 2 \mathrm{arcmin}$ & $\pm 20 \mathrm{um}$ \\
\hline Folding Flat 1 & $\pm 100 \mathrm{um}$ & $\pm 2 \mathrm{arcmin}$ & $\pm 100 \mathrm{um}$ \\
\hline Grating & $\pm 100 \mathrm{um}$ & $\pm 2 \mathrm{arcmin}$ & $\pm 100 \mathrm{um}$ \\
\hline Folding Flat 2 & $\pm 100 \mathrm{um}$ & \pm 2 arcmin & $\pm 100 \mathrm{um}$ \\
\hline Schmidt Mirror 2 & $\pm 100 \mathrm{um}$ & \pm 2 arcmin & $\pm 100 \mathrm{um}$ \\
& \multicolumn{3}{|c}{ Optical alignment if needed } \\
\hline Detector & \multicolumn{3}{|c}{ Optical alignment }
\end{tabular}

Table 3: Schmidt collimator and camera alignment tolerances

\section{A 2400 G/MM UV SCHMIDT GRATING AT THE HEART OF THE SPECTROGRAPH}

The collimator and the camera described above share a reflective $2400 \mathrm{~g} / \mathrm{mm}$ aspherical grating manufactured by JobinYvon using a technique developed by G.R. Lemaitre ${ }^{4}$. This technique has been used for several ground and space spectrographs ${ }^{5}$ where the disperser is a reflective axisymmetric Schmidt grating. The particularity of this technique is to produce an aspherized grating via two replica stages of a plane grating by use of an intermediate active optics metallic 
matrix. Starting from a plane diffraction grating known as the master, the first replication is performed on the plane surface of the unstressed matrix. In a second stage the deformable matrix is aspherized by applying the right air pressure and then replicated on the final slightly concave Zerodur substrate of the grating. The radius of the grating blank is chosen to minimize the thickness of the replica resin layer.

We control the aspherization of the flight grating (Figure 5 left) in front of an interferometer using a converging lens to introduce spherical aberration and then reduce the number of fringes due to the large departure of the grating shape from a plane. Figure 5 shows the good agreement between the measured interferogram (middle) and the one simulated (right) under Zemax with the setup parameters and theoretical aspherization coefficients of the grating.

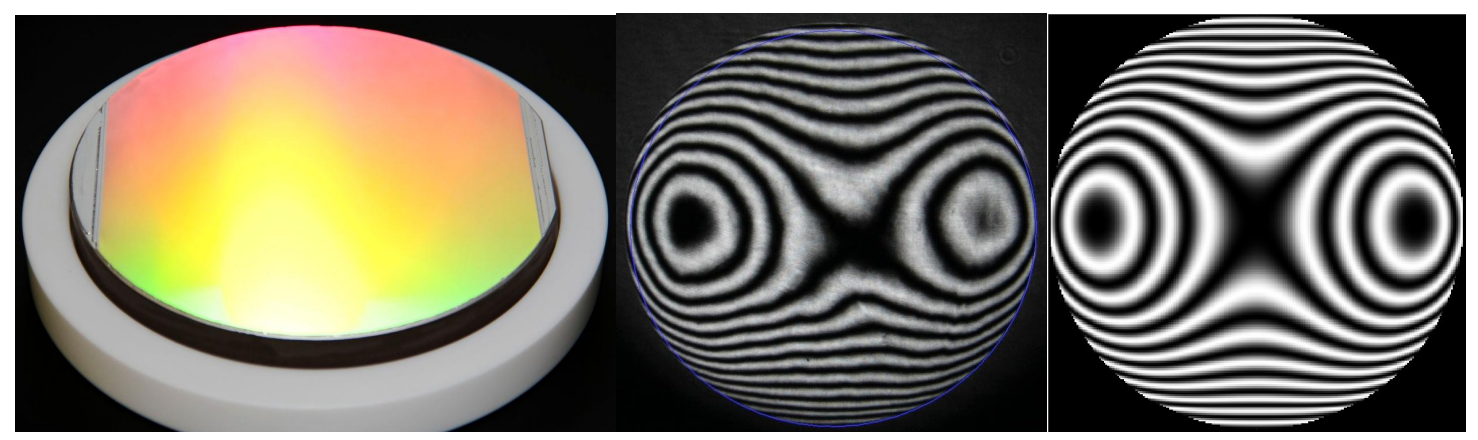

Figure 5: (left) Flight grating, (middle) Measured interferogram, (right) Zemax interferogram

Since the grating efficiency plays a key role in the throughput of the instrument, we developed ${ }^{6}$ a PCGrate model of the grating from polarized measurements at $12^{\circ}$ deviation angle in the range $200-350 \mathrm{~nm}$ of a $50 \times 50 \mathrm{~mm}$ replica of the same master used for Fireball. The measurement at $12^{\circ}$ deviation angle comes from a limitation in our commercial spectrophotometer. The PCGrate model is used to predict the efficiency of the grating at its $28^{\circ}$ working deviation angle and then to specify to Jobin-Yvon the optimum thickness of the aluminum coating. Figure 6 (left) shows the flight efficiency measurements at $12^{\circ}$ deviation angle and the model with a $25 \mathrm{~nm}$ aluminum coating to match the measures in $\mathrm{P}$ - polarization. For the $28^{\circ}$ deviation angle of the design (figure 6 right), this model predicts at $205 \mathrm{~nm}$ an efficiency of $62 \%$ (S-polarization) and 57\% (P-polarization) for an aluminum coated grating with an $\mathrm{Al} 2 \mathrm{O} 3$ natural oxidation layer.
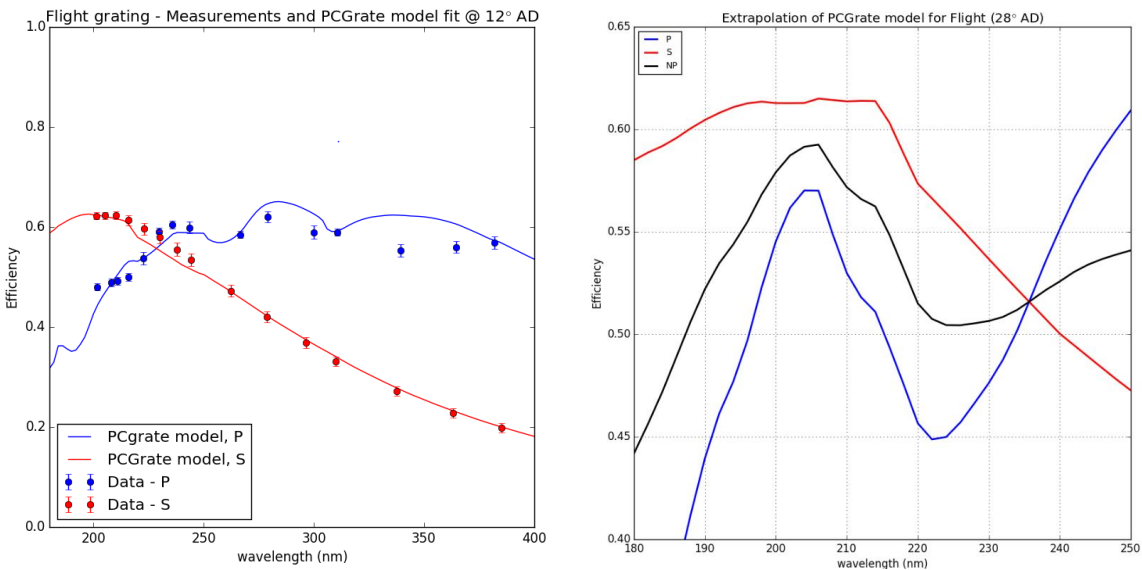

Figure 6: (left) Efficiency measurements of the flight grating at $12^{\circ}$ deviation angle. (right) Model prediction at $28^{\circ}$ working deviation angle

\section{UV ZINC LAMP SPECTRA AS A TEST OF THE SPECTROGRAPH}

At the center of its field, the focal corrector is stigmatic and so it can be used without the parabola. To test the spatial and spectral resolution of the spectrograph, we illuminated a diffuser with a Zinc lamp and a $10 \mu \mathrm{m}$ pinhole at the entrance of 
the field corrector. At the slit level we chose an open mask to not block the light. The detector was an R grade UV CCD developed by Caltech/JPL for test purposes. We shimmed the 3 attachment points of the pinhole outside the vacuum tank to optimize the focalization. All this test was performed under vacuum with a detector working at $140 \mathrm{~K}$.

Figure 7 shows the spectra of three Zinc lamp lines $(213.9 \mathrm{~nm}, 206.2 \mathrm{~nm}, 202.5 \mathrm{~nm})$ and a zoom on the $202.2 \mathrm{~nm}$ with a measured FWHM of 32 micrometers. This spectra allows to deduce the spectrograph plate scale. The $11.4 \mathrm{~nm}$ bandpass extends over $6.86 \mathrm{~mm}$ giving a plate scale of $1.66 \mathrm{~nm} / \mathrm{mm}$. Then a 50 micrometers slit width corresponds to a delta wavelength of $0.083 \mathrm{~nm}$ and, assuming the case of uniformly illuminated slit the spectral resolution, would be around $\mathrm{R}=2300$ as expected.
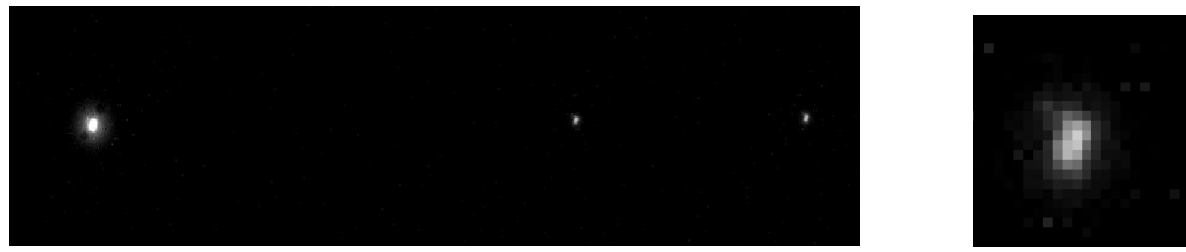

Figure 7: Zinc lamp Spectra showing three lines (213.9 nm, 206.2nm, 202.5nm) in the Fireball bandpass (left). Zoom on the $202.5 \mathrm{~nm}$ line; Detector pixel size is 13 micrometers (right)

\section{CONCLUSION}

The Fireball spectrograph optics and, in particular, its challenging unit magnification focal corrector has been manufactured within the specifications. To achieve tight alignment tolerances of the focal corrector we used a liquid shim technique by bonding in situ the small convex while the PSF was checked. The Schmidt grating, a key component of the spectrograph, was manufactured using active optics to achieve highly non symmetric aspherization. A careful optimization of its efficiency based on sample measurements and model prediction allow to reach an efficiency of 59\% at $205 \mathrm{~nm}$ for unpolarized light. Finally, feeding the payload with an UV Zinc lamp allows us to produce spectra onto the engineering detector and validate the spatial and spectral resolution of the spectrograph.

During the integration of the payload at LAM, we discovered in April this year that three spectrograph mirrors coated with Aluminum plus MgF2 had poor efficiency $(60 \%)$ compared to the samples $(92 \%)$ evaporated in the same batch. The resulting 3.5 efficiency degradation precludes a flight in the fall of 2016 and a recovery plan is under study for a 2017 flight from Fort Sumner.

\section{REFERENCES}

[1] Montel, J. et al, "Design and Improvements of the Attitude Control System of the FIREBall balloon experiment", Space Telescopes and Instrumentation 2016: Ultraviolet to Gamma Ray, [9905-164], Proc. SPIE 9905, (2016).

[2] Grange R., Milliard B., Mclean R., Martin C., Deharveng J.M., Schmininovich D., "Three-Dimensional Spectroscopy with a fiber-fed NUV Spectrograph" Proc. SPIE 5898 (2005).

[3] Grange, R. et al, "Multi object spectrograph of the FIREBall balloon experiment", Space Telescopes and Instrumentation 2014: Ultraviolet to Gamma Ray, [9144-30], Proc. SPIE 9144, (2014).

[4] Lemaitre G.R., [Astronomical Optics and Elasticity Theory], Springer, 217-261 (2001).

[5] Lemaitre G.R., Richardson E.H., "Ground based and Orbital off-axis aspherized grating imager-spectrographs: ISARD/Pic du midi and OSIRIS/Odin-Orbiter", Proc. SPIE 3355, 682-695 (1998).

[6] Quiret, S. et al, "The FIREBall-2 UV sample grating efficiency at 200-208nm", Space Telescopes and Instrumentation 2014: Ultraviolet to Gamma Ray, [9144-30], Proc. SPIE 9144, (2014). 